\title{
Reference Frames in Astronomy
}

\author{
K. J. Johnston \\ U.S. Naval Observatory, 3450 Massachusetts Ave. NW, Washington, D.C. 20392-5420; \\ e-mail: kjj@astro.usno.navy.mil
}

Chr. de Vegt

Hamburger Sternwarte, Gojenbergsweg 112, 21029 Hamburg, Federal Republic

of Germany

Key Words astrometry, reference frames, reference systems

- Abstract Advances in wide-angle astrometric measurements of three to four orders of magnitude in the last thirty years have resulted in a redefinition of the fundamental astronomical reference frame. This new frame, the International Celestial Reference Frame (ICRF), is based on the radio positions of 212 compact extragalactic radio sources. The ICRF defines the direction of the axes of the International Celestial Reference System (ICRS) with a precision of approximately $20 \mu$ as. At optical wavelengths, the Hipparcos catalog is the realization of this frame. The precision with which the ICRF is now determined requires that the ICRS models for precession, nutation, and others, be revised. Increases in the precision of measurements from astrometric space missions will further improve the celestial reference frame and may require its redefinition within the next ten years. These improvements will again challenge the models for the celestial reference system.

\section{INTRODUCTION}

Reference frames used in astronomy are defined by the positions of objects on the celestial sphere, usually specified only by direction. Astrometric advances provided by Very Long Baseline Interferometry (VLBI), radar ranging to the inner planets, laser ranging to the moon, and the European Space Agency (ESA) Hipparcos Space Astrometry mission have improved the determination of the position of celestial objects by three to four orders of magnitude. Figure 1 shows these advances in the context of achievements in astrometry since 150 B.C. Increasing accuracy dictates that relativistic effects (which are of order $10^{-8}$, and are at the milliarcsecond or mas level for angles of order of a radian) be taken into account. Further, the distances of some of these objects (i.e. extragalactic radio sources) are so large that kinematic motions of these objects do not contribute to apparent temporal positional changes greater than a few microarcseconds ( $\mu$ as). According to Mach's principle, there are no fixed (inertial) reference points for the frame because all 


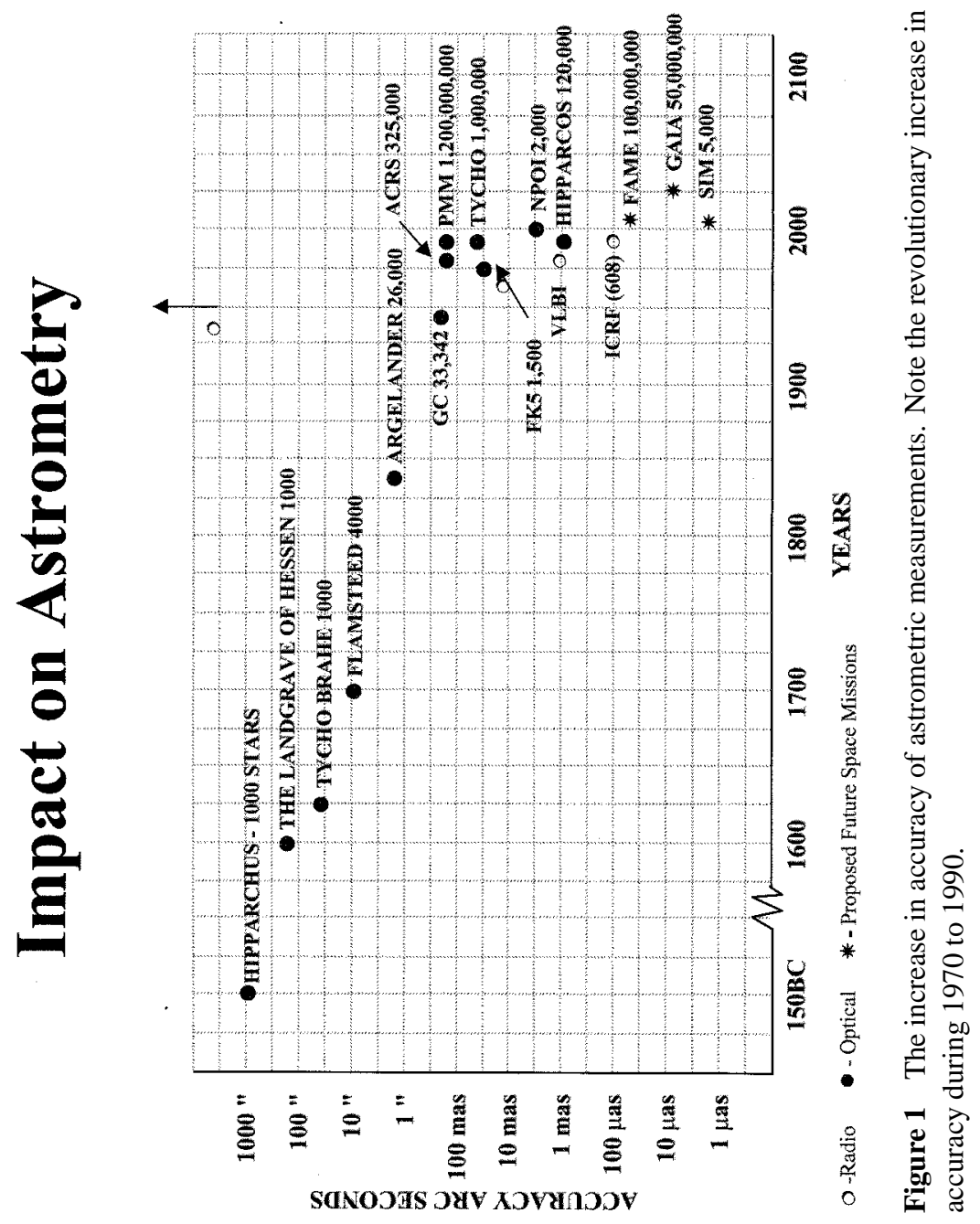


objects in the universe interact with one another. The positions of these objects then define a quasi-inertial reference frame. Previously the positions of nearby bright stars had been employed in this capacity. The system's zero point was based upon the motions of the Earth and thus was a dynamical/kinematic reference system. The system defined now by the extragalactic radio sources is a purely kinematic system.

At the XXIII IAU General Assembly in 1997, the new ICRS and its realization, the ICRF, were adopted to replace the traditional optical fundamental reference system as realized by the FK5 reference frame. The ICRF is based exclusively on the directions to selected strong compact extragalactic radio sources determined by VLBI techniques. The IAU has further resolved that the realization of the radio reference frame in the optical domain shall be primarily the high-precision stellar net of the 118,218 stars constructed from the ESA Hipparcos Space Astrometry mission. With this development, the optical and radio regions of the spectrum are now related at the mas level.

Previous Annual Reviews articles associated with this topic have addressed radio astrometry (Counselman 1978) and astrometry (van Altena 1983, Monet 1988, Kovalevsky 1998). Other reviews in the field of astrometry and reference frames may be found in Sovers et al (1998); Vondrak \& Capitaine (1998); Kovalevsky et al (1989); IAU Colloquium 127, Reference Systems, edited by Hughes, Smith, \& Kaplan (1991); IAU Symposium 166, Astronomical and Astrophysical Objectives of Sub-millarcsecond Optical Astrometry, edited by Hog \& Seidelmann (1995); IAU Symposium 172, Dynamics, Ephemerides and Astrometry of the Solar System edited by Ferraz-Mello et al (1995); IAU Colloquium 165, Dynamics and Astrometry of Natural and Artificial Celestial Bodies, edited by Wytrzyszczak et al (1996); and the International Earth Rotation Service (IERS) Conventions (IERS 1996), http://maia.usno.navy.mil/conventions.html.

A basic overall reference is the Explanatory Supplement to the Astronomical Almanac (Seidelmann 1992) and references therein. Radio astronomy and astrometry techniques are dealt with in Meeks (1976), Thompson et al (1986), and Zensus et al (1995). The Hipparcos mission is described in a 17-volume set (ESA 1997).

With the refinement of radio interferometric techniques for astrometry, especially VLBI, and the success of the Hipparcos mission, research efforts have intensified during the past 30 years. This review presents the background for the adoption of the ICRF, its realization at optical and radio frequencies, its limitations, and future outlook for increased precision. The references mentioned here deal in great detail with reference systems and reference frames as well as optical and radio astrometry, which are the basis for the adoption of the ICRF.

\section{PRINCIPLES OF CELESTIAL REFERENCE SYSTEMS AND REFERENCE FRAMES}

The reference system defines the coordinate system by specifying the direction of the axes, and specifying the zero points. The reference frame is defined by the positions of objects along with their coordinates in the system and is the practical 
realization of the reference system. In principle, two fixed points can define the reference frame, allowing the reference system to define its principal plane, pole, and zero point. In practice, many fixed points are used to define the reference frame globally because the definition of the axes of the system improves with the number of points defined. Also many fiducial points are needed to improve the ability to access the reference system anywhere on the celestial sphere.

In view of the high precision achieved by modern astrometric measuring methods from ground and space, and considering that those in the future will approach the microarcsecond level, a clear, rigorous specification of terms is required so that inconsistencies are not introduced into the definitions of the reference system and reference frame. Following the terminology introduced by Kovalevsky \& Mueller (1981) (see also Kovalevsky et al 1989 for a detailed discussion) the purpose of a reference frame is to provide the means to materialize a reference system so that it can be used for a quantitative description of the positions and motions of celestial bodies.

A reference system is the underlying theoretical concept for the construction of the corresponding frame. An ideal celestial reference system, then, would be an inertial (i.e. nonrotating) system in the Newtonian definition which, however, is applicable only locally in General Relativity. The actual construction of a reference system requires the identification of a physical system to which the ideal reference frame definition is applied. Such a choice is naturally not unique, but the following two definitions are suitable.

In an ideal dynamical reference system, the equations of motion of a celestial body do not contain any rotational or acceleration terms: an inertial system in the Newtonian definition of a universal reference system and applicable only locally in General Relativity, where the transport of a coordinate system from one point to another is a complex transformation involving the exact knowledge of the mass distribution everywhere in that part of the space involved. (For a detailed discussion see Kovalevsky et al 1989, Seidelmann 1992, and Soffel 1989). Because of the local restriction of a given ideal reference system, the term "quasi-inertial system" is used.

In an ideal kinematic reference system, it is assumed that the universe does not rotate. Thus a suitable class of extragalactic objects, such as quasars or remote galaxies, do not display any group rotation. The ideal kinematic reference system is essentially based on the kinematic properties of very distant objects, which are assumed to be random. In Newtonian mechanics these different reference system definitions are equivalent. In General Relativity the local character of any reference system must be taken into account.

The actual modeling of this ideal reference system concept will depend on the numerical values of a number of parameters, which, as a result of observations, are not known exactly and must be adopted. Therefore the model is only an approximation of the ideal situation and is called a conventional reference system.

For centuries the primary astronomical reference systems and frames were restricted to the optical spectral region based on the continuous observations of a small number of bright fundamental stars and members of the solar system. These 
data resulted in a series of fundamental star catalogs, the latest being the Fifth Fundamental Catalog (FK5) (Fricke et al 1988). In the radio domain, the now mature and highly precise VLBI observing technique has been used to define and maintain a celestial (and make a contribution to the terrestrial) coordinate system with sub-mas precision which now supersedes the optical systems precision by at least one order of magnitude.

Following the recommendations of the IAU working group on Reference Systems (Hughes et al 1991, Proc. IAU Coll. 127), in 1991 the IAU decided that the future IAU conventional celestial reference system should be based on a set of distant extragalactic objects with no global rotation (Bergeron 1992). A list of suitable candidate objects (mainly QSOs, AGNs, and compact extragalactic radio sources) to define the associated new conventional reference frame was adopted (Appenzeller 1994). The XXIII General Assembly 1997 (Bergeron 1997) approved the total concept, resolving that (a) from January 1, 1998 the IAU celestial reference system shall be the International Celestial Reference System (ICRS) as specified by the 1991 IAU resolutions and as defined by the International Earth Rotation Service (IERS) (see Arias et al 1995, IERS 1997); (b) the corresponding fundamental celestial reference frame shall be the International Celestial Reference Frame (ICRF) constructed by the IAU Working Group on Reference Frames (WGRF); (c) the Hipparcos Catalog shall be the primary realization of the ICRF at optical wavelengths; $(d)$ IERS should take appropriate measures, in conjunction with WGRF, to maintain the ICRF and its ties to the reference frames at other wavelengths.

According to the IAU resolutions, the origin of the ICRS coordinate axes is at the barycenter of the Solar System. To achieve this condition, all observations are modeled within the framework of General Relativity. The directions of the axes are fixed with respect to the extragalactic sources. To maintain continuity with the FK5 System, the principal plane of the new system is kept close to the mean equator at $\mathrm{J} 2000.0$ and the direction of the new conventional pole is held consistent within the errors of the FK5 system. The origin of right ascension of the new reference system will be close to the dynamical equinox at J2000.0. With reference to the definitions discussed here, the ICRS is the realization of a conventional kinematic quasi-inertial reference system. The corresponding frame (Section 6) is materialized by a catalog of extragalactic source positions.

The conventional dynamical reference system is based on the theory of the motions of the bodies of the solar system and is constructed in such a way that there are no rotational terms in the equations of motion. Thus the corresponding dynamical reference frame (Section 4) is based on the specific choice of solarsystem ephemerides, the origin of the frame, and the adopted set of fundamental constants (masses of planets and auxiliary constants).

The fundamental reference system of observational optical astronomy has been based for the last 100 years on a net of selected bright stars, the so-called fundamental stars, covering the whole sphere somewhat uniformly. The term "fundamental" refers to the adopted observational procedures-that is, "fundamental 
observations"- that allow the construction of a net of star positions at a specified epoch without reference to previous determinations. The materialization of the system is achieved by a subsequent series of fundamental catalogs, containing the positions and proper motions of these fundamental stars together with a set of fundamental constants, in particular an adopted precession constant and nutation theory.

This conventional celestial reference system (CCRS) is modeled by these basic structures: ( $a$ ) the origin of the CCRS (barycenter of the solar system), $(b)$ the fundamental plane (celestial equator), and $(c)$ the zero point of the fundamental plane (vernal equinox, intersection of mean equator and ecliptic). The actual procedures for establishing a fundamental star system have been modified continuously over time depending on available instrumentation and accuracy requirements. However, two basic operations were used throughout: measuring the positions of stars relative to one another, and determining the position of the pole and the plane of the equator and the equinox with respect to these stars by observing the apparent motions of the Sun and selected planets relative to them. Until recently, the precision with which these measurements were made over large angles was 0.1 arcseconds with a transit circle. The celestial reference system must have a reference epoch and specify all the necessary procedures and constants required to transform the frame from the reference epoch to any other date. These procedures involve precession, nutation, space motion, parallax, aberration, and light deflection, as well as those local to the observer: earth orientation parameters, refraction, and time.

\section{THE FK5 REFEREN CE FRAME}

The CCRS was materialized by a consecutive sequence of fundamental catalogs, the most important being the FK-series of Fundamental Catalogs of the Astronomische Rechen-Institut in Germany. These catalogs were a compendium based on catalogs containing fundamental observations, meaning that the positions were on an instrumental system, the pole was determined independently, and the zero point of right ascension was adjusted to a dynamical system via observation of the sun and Solar System objects. These fundamental catalogs contained three major elements: positions, proper motions, and an adopted value for precession in order to define the fundamental coordinate system at its initial epoch, which then could be projected to other epochs and equinoxes.

Newcomb (1905a) published a catalog of 1257 stars with positions reduced to epochs 1875 and 1900 using a precession constant he derived from fundamental stellar proper motions (Newcomb 1905b). Boss (1937) published the General Catalog (GC), a catalog containing 33,342 stars from 238 catalogs obtained from observations as early as 1777 .

The Dritter Fundamental-Katalog des Berliner Astronomischen Jahrbuchs (FK3) (Kopff 1937) based on the FC and NFK catalogs originated by Auwers (1879), contains stars with long and good observational histories, which limited the number of entries in the FK3 (Part I) to 925 stars. 
The FK3 and FK4 catalogs were in use during most of the twentieth century. The FK3 is a realization of the dynamical reference system based on Newcomb's value of precession and a dynamical theory of motion in the solar system. The FK4 (Fricke \& Kopff 1963) is essentially an improvement of FK3 (improved star positions and proper motions removing regional errors only). Thus the system of the FK3 was still represented. (For details, see Woolard \& Clemence 1966, Mueller 1969, Eichhorn 1974).

Because Newcomb's determination of precession is also based on the analysis of the proper motion of fundamental stars and a model of local solar motion, all fundamental catalogs contain aspects of a kinematic system.

With the last catalog in this series, the FK5 (Fricke et al 1988), major changes and improvements were introduced. The FK5 system uses the IAU 1976 value of precession (Lieske et al 1977) and nutation (Seidelmann 1982), a new determination of the equinox and equator (Fricke 1982), a precessional correction determined from FK4 proper motion assuming a kinematic model of parallactic motion and galactic rotation (Fricke 1981) and sidereal time correction (Aoki et al 1982). The equinox of the accepted dynamical ephemerides (DE200/LE200) was made to agree with the catalog equinox of the FK5 (Standish 1982).

This implies that the FK5 system is not a pure dynamical system but partly kinematic (see also Kovalevsky et al 1989). The FK5 at epoch approximates an inertial reference frame related to the dynamical equinox as right ascension zero point.

The FK5 contains 1535 primary (FK5 I, Fricke et al 1988) and 3117 additional bright fundamental stars (FK5 II, Extension, Fricke et al 1991). The mean precision of the FK5 catalog positions and proper motions at average mean epoch 1950 is \pm 0.02 arcsecond and $\pm 0.8 \mathrm{mas} / \mathrm{yr}$, respectively. The quality of the FK5 frame is time dependent and is decreasing continuously by propagation of systematic and random proper motion errors, which introduce regional distortions at different epochs. A detailed comparison of the FK5 frame with the Hipparcos frame (Section 8) at epoch 1991.25 has shown large regional distortions up to 150 mas (Mignard \& Froeschle 1997). However, the inherent high quality of the basic FK5 I proper motions due to their large time basis will be used together with the Hipparcos data in order to obtain improved proper motions in particular for unresolved astrometric binaries (FK6 project, Wielen et al 1997).

\section{THE DYNAMICAL REFERENCE FRAME}

The Dynamical Reference Frame is defined by the motions of objects in the solar system. The standard system makes use of the equator of the Earth and equinox defined by the intersection of the mean plane of the equator and ecliptic. This reference frame is defined by the process of calculating the ephemerides of solar system bodies using the equations of motion in the chosen frame, adjusting these calculated positions to the frame of observation and observed positions of these 
bodies. In this way these computational ephemerides are then consistent with the observational data. When sufficiently accurate observational data are available, this fitting procedure can provide the constants necessary for the ephemerides.

Range measurements and spacecraft observations are independent of any external frame but have a strong dependence on observing sites and an accurate basis for time. These observations refer the position of the planet or moon with respect to the orbit of the Earth. Other measurements such as the optical positions of the planets are dependent on the stellar reference frame. The accuracies of the range and spacecraft measurements are by far superior to those of the optical. For example, radar ranging measurements to the planets have accuracies of $2 \mathrm{~km}$ to $100 \mathrm{~m}$, which correspond to a precision of $10^{-8}$ to $10^{-9}$, while the optical transit circle observations are precise at the $10^{-6}$ level. Jet Propulsion Laboratory's ephemerides before DE130 were oriented by the optical observations, which were on the FK4 reference frame.

Beginning with the DE200 ephemeris, JPL attempted to put the ephemerides onto the mean equator and equinox of J2000 (Standish 1982), but this was not strictly possible because of the uncertainty in the definition of the ecliptic (Standish 1981), the accuracy of the optical observations of the planets were poor, and the precession of the equator from mean epoch to J2000 was inaccurate by $0.3^{\prime \prime} /$ century. Although these ephemerides were on the accepted celestial system in use, they did not combine observations of the inner planets and moon-whose positions were determined very accurately by ranging and spacecraft measurement-with those of the outer planets, which are dominated by measurements in the accepted celestial reference frame. This resulted, effectively, in two frames: one for the inner planets and one for the outer planets.

\section{THE RADIO REFERENCE FRAME}

With the evolution of radio interferometry from 1950 to 1985, two major developments occurred. Intense, nonthermal, compact radio sources were discovered (Allen et al 1962a,b, Palmer 1962), and later identified as the extragalactic source known as quasars (Matthews \& Sandage 1963, Schmidt 1963, Oke 1963). Interferometric techniques were developed for locating the positions and determining the spatial sizes of discrete radio sources (Bolton \& Stanley 1948, Ryle \& Smith 1948), resulting in the association of the radio source Cygnus A with a distant galaxy (Baade \& Minkowski 1954). The accuracy of the position was ten seconds of arc in right ascension and forty seconds in declination. Radio interferometry evolved into linear arrays such as the Cambridge Array (Ryle 1972) and the Green Bank Interferometer (Hogg et al 1969) which was the prototype for the Very Large Array (Hjellming \& Bignell 1982). The antennas in these arrays were connected via cables. In the 1960s Very Long Baseline Interferometry (VLBI), using independent local oscillators to link telescopes that were not physically connected via cable or radio link, was demonstrated (Carr et al 1965, Bare et al 1967, Broten et al 1967, Moran et al 1967). 
The positions of compact radio sources were determined to less than $1^{\prime \prime}$ by Wade (1970) and Brosche et al (1973), and refined to 0.02 (Wade \& Johnston 1977) using the Green Bank Interferometer. This was paralleled by accuracies achieved via VLBI: 1" (Cohen \& Shaffer 1971) and 0.02 (Clark et al 1976). At this point the accuracy of radio positions surpassed that of optical transit circles.

In 1978 at the IAU Symposium 182, Time and the Earth's Rotation, an IAU Working Group was formed to promote the comparison and evaluation of techniques for the determination of Earth rotation and high-precision data for scientific analysis. The efforts of the IAU Working Group later became known as Project MERIT ("MERIT" is an acronym for "Monitor Earth Rotation and Intercompare Techniques"). During the 1980s Project MERIT carried out many campaigns evaluating laser ranging and VLBI techniques, and adopted standards for obtaining the highest precision of measurements (Melbourne et al 1983). Project MERIT's efforts led to the establishment of the International Earth Rotation Service (IERS) on January 1, 1988. The IERS replaced the Bureau International de l'Heure and the International Polar Motion Service, and serves as a general coordinating agency for the development of reference frames and the relationship of geodetic and astronomical methods for precise measurements. The IERS adopted conventions for the celestial, dynamical, and terrestrial reference systems and frames, as well as numerical standards (precession, masses of the planets, etc), and models for nutation, tropospheric refractivity, geopotential, site displacement, etc. These conventions are updated as often as necessary and were most recently published in the IERS Technical Note 21 (1996).

By 1978, the promise of improved accuracy was about to be fulfilled. At IAU Colloquium 48, Modern Astrometry, a working group was established under IAU Commission 24 to select candidate radio sources with optical counterparts for a reference frame. The working group identified 234 candidate sources, the majority of which had positional accuracies of 10 mas. The positions of these sources were obtained by a weighted average of eight catalogs, seven of which were obtained with interferometers connected with cables (Argue et al 1984).

In the 1980s great advances were made using VLBI techniques. Observations were standardized. Two frequencies, 2.3 (S-band) and $8.4 \mathrm{GHz}$ (X-band) generally using fourteen channels of two MHz bandwidth each were used. Six S-band channels spanned about $85 \mathrm{MHz}$ while eight X-band channels spanned about 360 MHz. The basic observable is group delay (Rogers 1970). The two frequencies allow for accurate calibration of the frequency-dependent propagation delay in the ionosphere. Catalogs of positional accuracy reached a few milliarcseconds (Fanselow et al 1984), and survey observations extended coverage to the Southern Hemisphere (Morabito et al 1986a,b) with accuracies of 300 mas.

The VLBI technique made a major advance with the introduction of the Mark III VLBI system (Rogers et al 1983, Clark et al 1985). Catalogs using Mark III technology achieved an accuracy of less than 0.5 mas for the best positions (Ma et al 1986, Robertson et al 1986); Mark II measurement by Sovers et al (1988) achieved the same level of accuracy. 
To obtain these accuracies, certain astronomical constants such as precession and nutation were solved from the data. The MERIT standards were brought forth to update the 1976 IAU standards (Melborne et al 1983). Two different software analysis systems evolved for the reduction of VLBI data: MASTERFIT (Sovers \& Fanselow 1987), developed by the Jet Propulsion Laboratory, and CALC /SOLVE/GLOBL, developed jointly by the Goddard Space Flight Center, the Harvard-Smithsonian Center for Astrophysics, and the National Geodetic Survey (Robertson 1975, Ma 1978, Gordon 1985, Ma et al 1986). Both sets of software were found to deviate in theoretical delays by 1.5 to 50 picoseconds, not including the comparison of the different tropospheric and relativistic models used by each (Sovers and Ma 1985).

Various catalogs of radio source positions were produced by JPL, GSFC, NGS, and USNO/NRL in the late 1980s. These catalogs were combined by the IERS to obtain a reference frame. By 1990, it was concluded that the accuracy of the source positions was better than 1 mas with regional deformations between catalogs of a few mas (Arias et al 1991). Later, the precision of source coordinates was found to be a function of the number of observations with an estimate of 0.2 mas for positions based on 100 observations (Arias et al 1995).

In 1986, a program to establish a global radio reference frame of 400 sources was undertaken (Johnston et al 1988). The resulting reference frame would be radio/optical: all the radio sources would have optical counterparts whose positions would also be measured. The program proposed to use all Mark III data available in a consistent solution (Johnston et al 1991), and resulted in a number of catalogs between 1991 and 1995. The first was a catalog of 182 sources with a positional accuracy of one mas (Ma et al 1990). The right ascension zero point was defined by the FK5 based optical positions of 28 quasars. These sources were all north of -30 degrees declination. This first program was followed by campaigns to increase the density of sources in the northern hemisphere and to add an equal grid of sources in the southern hemisphere (Russell et al 1991, 1992, 1994; Fey et al 1992, 1994; Reynolds et al 1994). These campaigns resulted in a catalog consisting of a total of 403 sources with 208 in the northern hemisphere and 195 in the southern hemisphere.

These data, together with all available dual-frequency bandwidth synthesis Mark III VLBI data from the geodetic and Earth orientation programs, were used to solve for catalog positions from first principles in a single solution (Johnston et al 1995). This data set consisted of all data (1,015,292 pairs of group delay and phase delay rate observations) collected between 1979 and 1993. The majority of the data were from the geodetic programs, Earth orientation programs, and source surveys.

The astrometric campaign contributed only 23,000 observations but filled out the reference frame especially in the southern hemisphere. It resulted in a catalog of 436 sources with positional accuracies better than three mas in each coordinate with the accuracies of the majority of sources smaller than one mas. These 436 sources defined the reference frame. The sources were divided into two classes. Class 1 sources (163 in northern hemisphere, 48 in southern hemisphere) have positional weighted rms accuracies of less than one mas, while class 2 sources (98 
in the northern hemisphere, 127 in the southern hemisphere) have weighted rms accuracies of less than three mas. Also measured were an additional 124 objects that needed further observation to improve their positions or were unsuitable for the reference frame because of complex source structure.

\section{THE INTERNATIONAL CELESTIAL REFERENCE FRAME}

As discussed in Section 2, in 1991 the XXI General Assembly of the IAU passed a resolution stating that the celestial reference system would be realized by a celestial reference frame defined by the precise coordinates of extragalactic radio sources. A working group on reference frames was established to create this catalog, which was accomplished in 1998. It includes primary sources, which define the frame, and secondary sources that later may be added to-or replace—-the defining sources. The criteria for the defining sources were sufficient data (observation for more than two years, with more than twenty observations) showing lack of position variation (differences less that 0.5 mas or $3 \sigma$ in either coordinate); have submilliarcsecond positional formal errors; a structure index (Fey \& Charlot 1997b) at X Band (if available) of one or two and show no significant apparent proper motion.

This work has been detailed by Ma et al (1998). A single solution of 1.6 million pairs of group delay and phase delay rates data obtained between August 1979 and July 1995 was made. A frame based on the positions of 212 defining extragalactic radio sources distributed over the entire sky has been established. The positional accuracy of these sources is less than one mas. The positions are at a frequency of $8.4 \mathrm{GHz}$, and were obtained from 1.6 million pairs of group and phase delay observations obtained by dual frequency 2.3 and $8.4 \mathrm{GHz}$ VLBI observations. Figure 2 shows the distribution of these sources on an Aitoff Equal Area

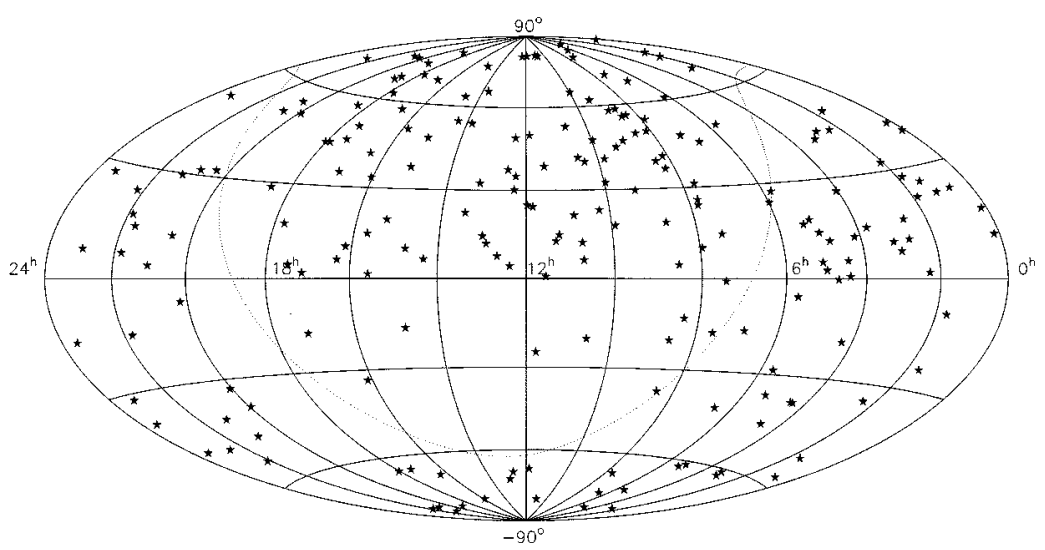

Figure 2 The distribution of ICRF defining sources. There is a lack of sources south of the equator due to lack of observations. A large number (more than 100) of observations are needed to obtain formal positional accuracy at the $100 \mu$ as level. 


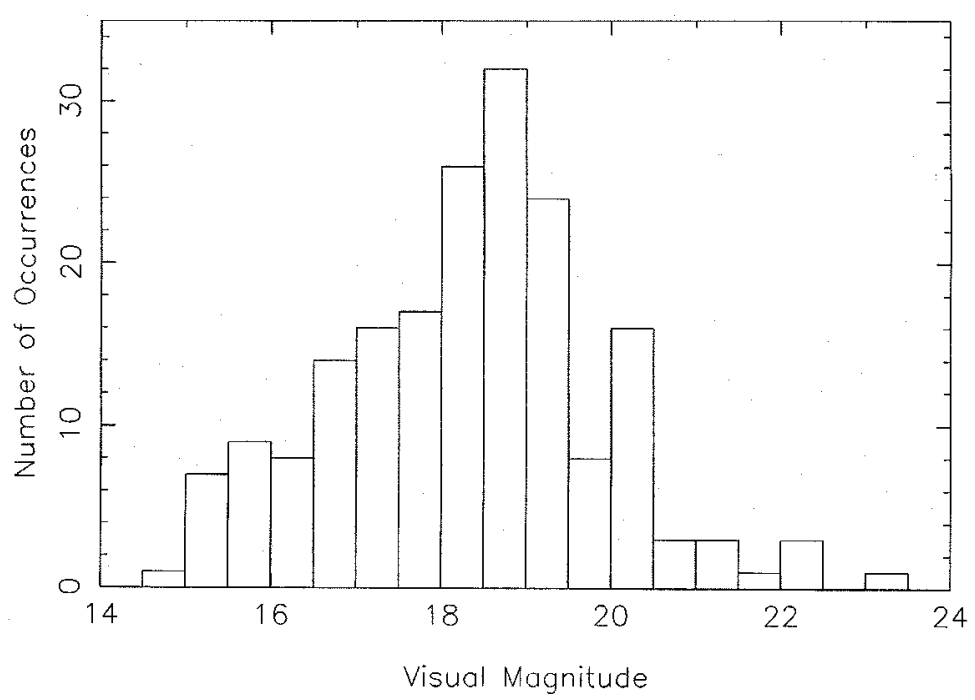

Figure 3 The visual magnitude of the optical counterparts of the ICRF sources. Note that they are much fainter than the Hipparcos stars and peak at about the $18^{\text {th }}$ visual magnitude. Thus bright stars with radio counterparts were used to link the ICRF and Hipparcos frames.

Projection. Only 22 percent of the defining sources are in the southern hemisphere, a fact attributable to a lack of VLBI observations: most radio observatories are in the northern hemisphere.

The accuracy of the positions of these sources is estimated by RSS, the formal error of the solution multiplied by 1.5 with a systematic error estimated to be 0.25 mas. As of January 1, 1998, the IAU adopted this frame as the fundamental reference frame. The optical magnitudes of the defining sources are presented in Figure 3. The majority of the sources have optical counterparts at the $18^{\text {th }}$ to $19^{\text {th }}$ visual magnitude. Through future observations, an additional 294 candidate sources may become defining sources. There are also 102 "other" sources whose positions may show variations with time, or whose positions are less well known.

\section{MAINTENANCE AND LIMITATIONS OF THE ICRF}

\subsection{Limitations D ue to Source Structure}

Extragalactic radio sources display structure on spatial scales from hundreds to one mas. The spatial structure is usually more compact at higher radio frequencies. The mechanism giving rise to these sources is believed to be the existence of massive 


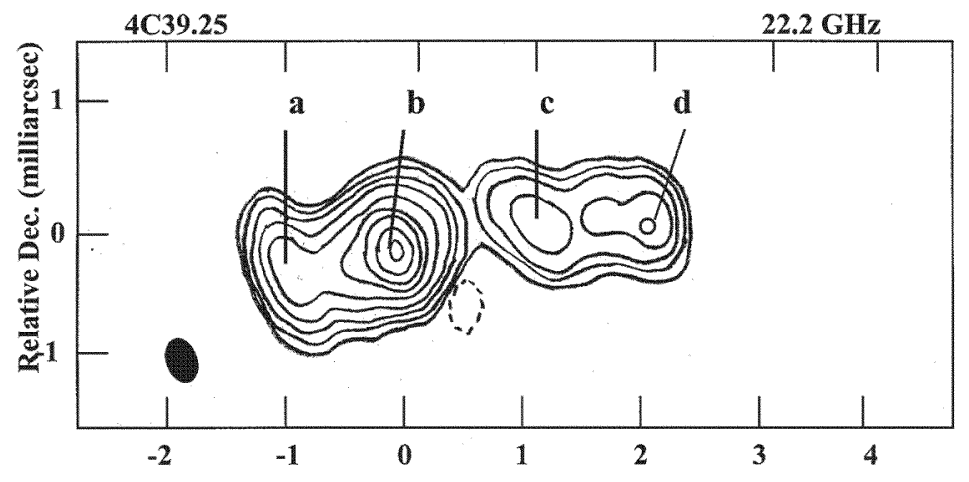

Relative R.A. (milliarcsec)

Figure 4 The distribution of flux density at $22 \mathrm{GHz}$ for the extragalactic radio source 4C39.25 (Guirado et al 1995). The restoring beam is shown as a filled ellipse. Note that $4 \mathrm{C} 39.25$ is distributed over 3 mas in the east-west direction.

black holes in the center of galaxies. The radio emission takes the form of jets. There is a wide variety of source structures. These sources are all variable on timescales of weeks to years at radio wavelengths. Recent observations have found timescales of hours for some of these objects.

Figure 4 presents typical source structure for one of these compact objects (4C39.25), obtained at a frequency of $22 \mathrm{GHz}$ (taken from Guirado et al 1995). Note that the source has at least four components distributed over about four mas in right ascension. The effect of variations in this structure is illustrated in Figure 5. A position for this source has been measured over a 15-year period using all geodetic Mark III VLBI observations. The solutions for right ascension and declination show variations due to changes in source structure with time. This source has an apparent proper motion of $13.6 \mu \mathrm{as} / \mathrm{yr}$ in right ascension and $6.8 \mu \mathrm{as} / \mathrm{yr}$ in declination (Fey et al 1997a), consistent with variations in components $\mathrm{a}$ and $\mathrm{b}$ identified in Figure 4. In the period 1980 through 1982 components $\mathrm{b}$ and $\mathrm{c}$ dominate the position. In the period 1982 through 1985 component a dominates, and in 1985 through 1997 component b dominates (Fey et al 1997a). Also note that the quality of the positions markedly improved after 1985. This improvement was probably due to the introduction of the Mark III system and improved observing techniques for geodetic measurements.

Maps of sources will also have different appearances when mapped with different arrays of antennas. Maps of the source VR422201 (BL Lac) at $8.4 \mathrm{GHz}$ are displayed in Figure 6. The top figure was obtained using the VLBA, and the bottom figure was made from the VLBA data with the addition of geodetic antennas. Note that the structure appears more compact when mapped with the VLBA plus geodetic antennas, which could result in a source position offset of a few tenths of a mas in declination. 

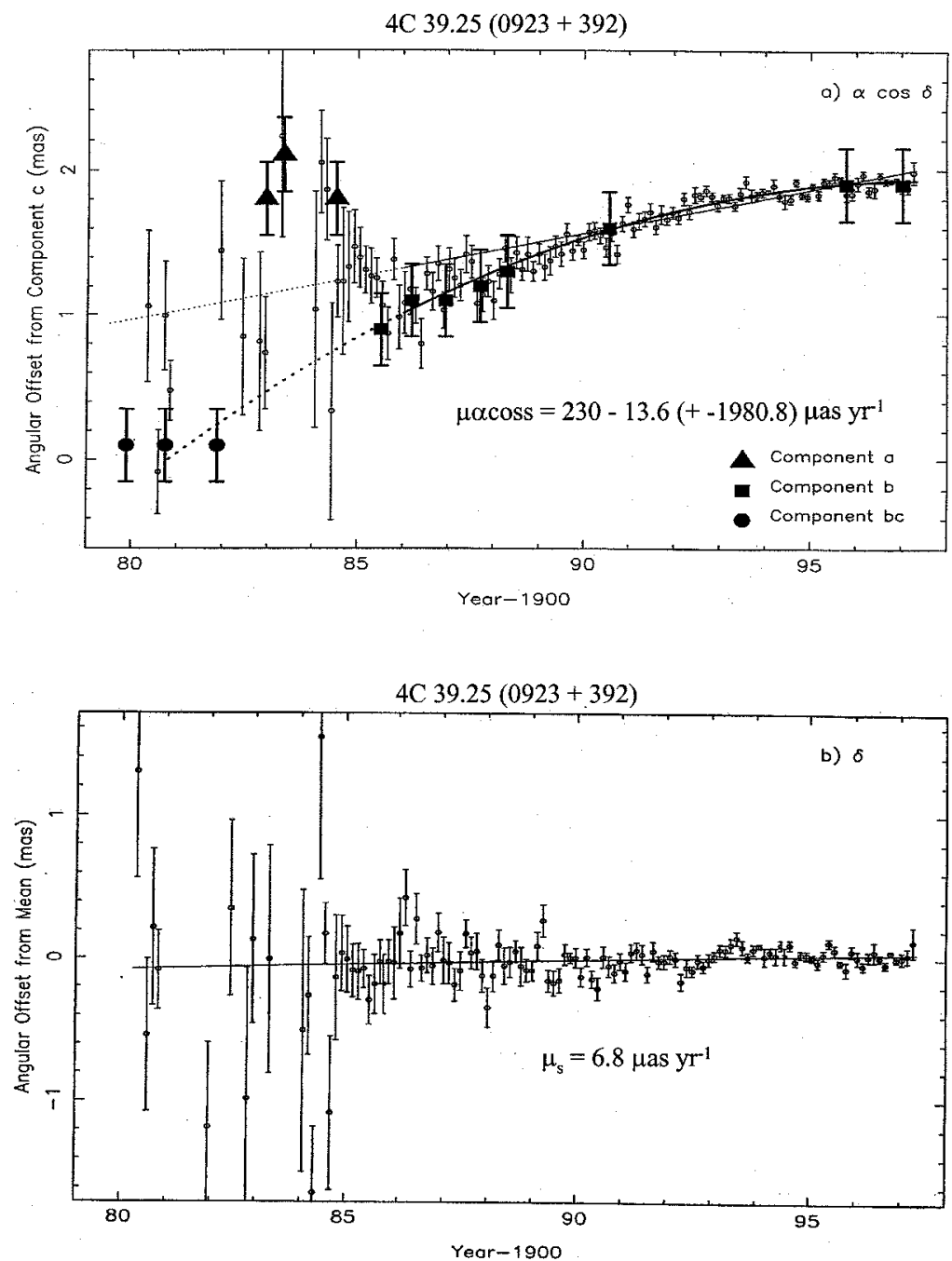

Figure 5 The position of 4C39.25 as measured with the geodetic database over the time period 1979-1997. There is significant apparent motion in right ascension, while the declination position is quite stable. Note that the quality of the data increased markedly after 1985 . The motion in right ascension is attributed to variations in the flux density of the components. 


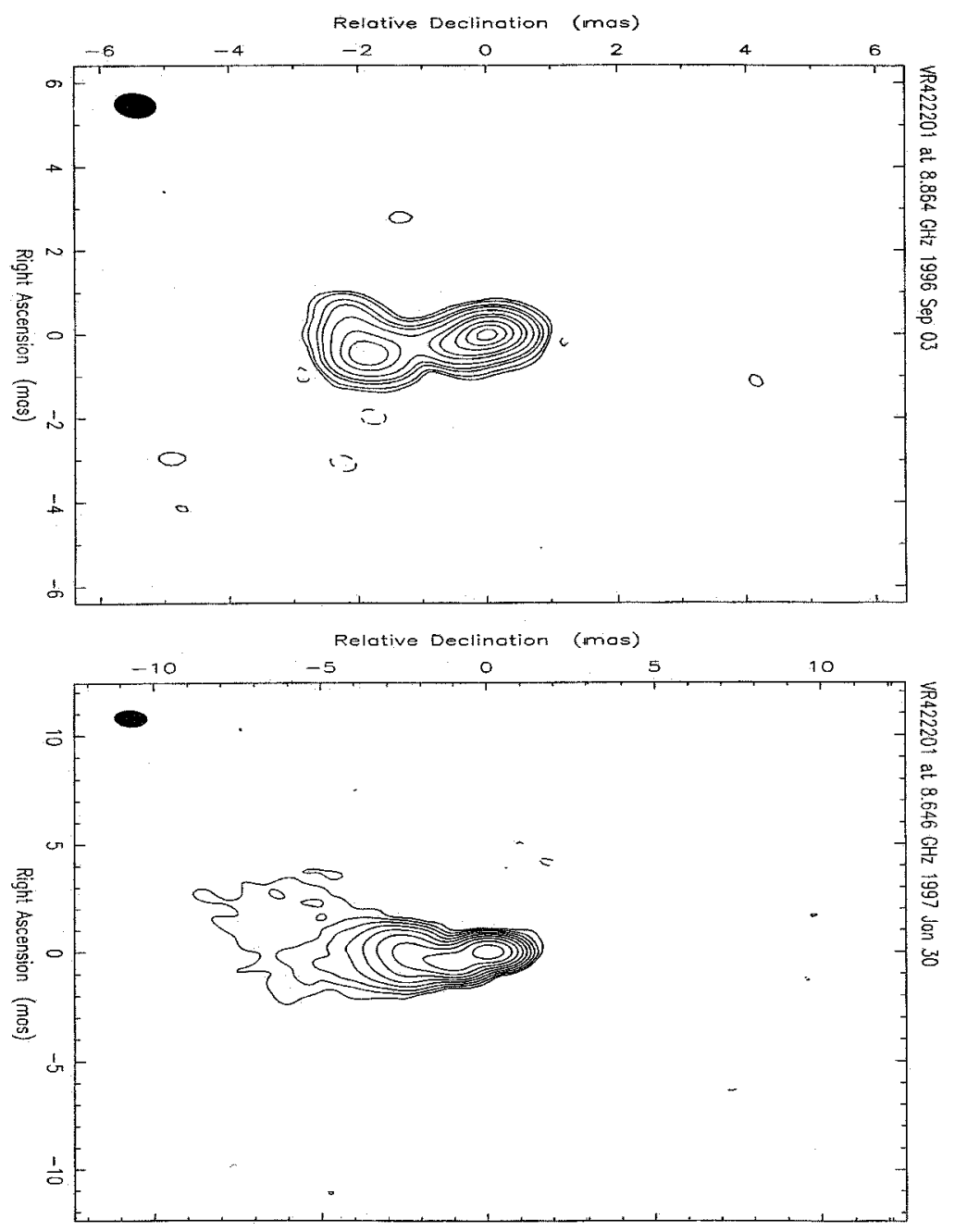

Figure 6 Maps showing the distribution of radio emission from the sources and VRO 42201 as mapped using the VLBA array (top) and the VLBA and geodetic antennas (bottom). The restoring beams are shown by filled ellipses. The emission is quite complex and appears slightly different as mapped by the different arrays.

The effect of source structure on position can be as large as tens of mas. From an investigation of source structure corrections to source positions, Fey \& Charlot (1997b) found a correlation between compactness of the sources and their formal positional uncertainties, indicating that more extended sources have larger positional uncertainties. They define a structure index to estimate the astrometric quality of the sources. An index of one at $8.4 \mathrm{GHz}$ is very good, two at $8.4 \mathrm{GHz}$ is good; three is marginal and should be used with caution and four should not be used. 

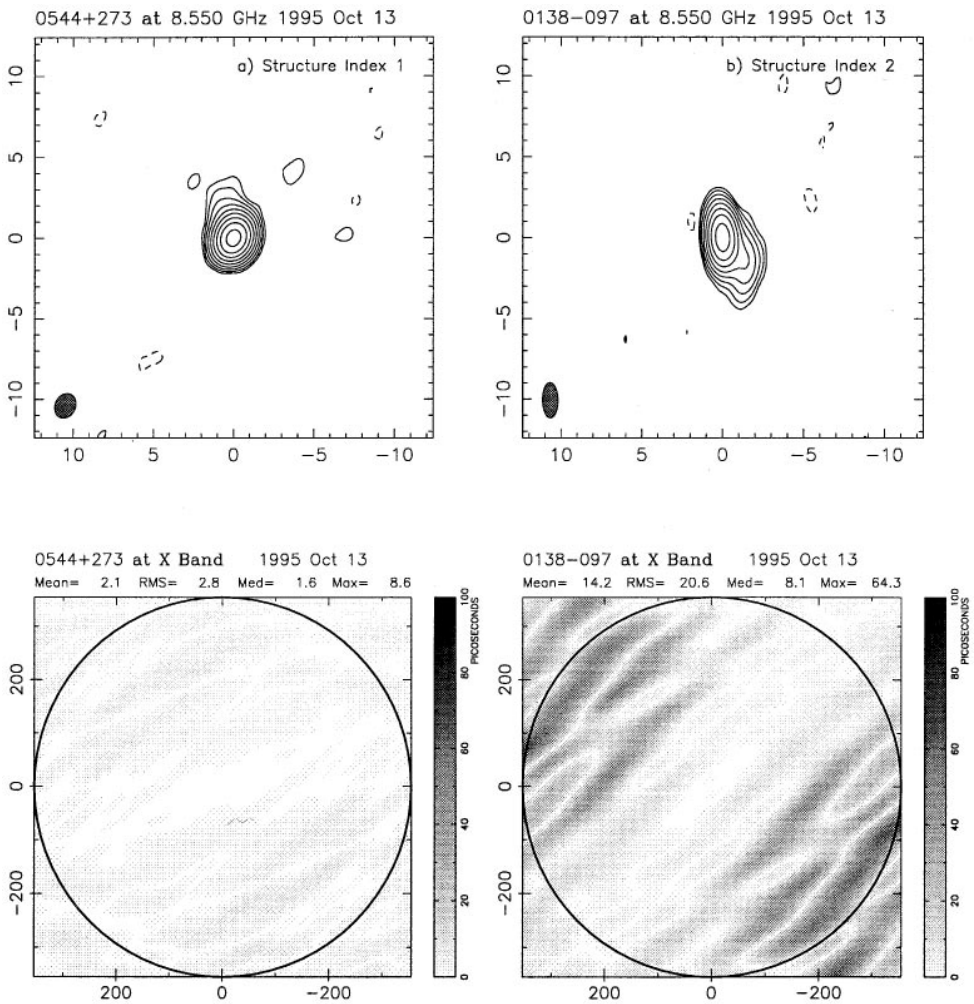

Figure 7 Structure contribution to measured interferometric delay. Top displays the $8.4 \mathrm{GHz}$ radio emission. Bottom shows the structure of the induced bandwidth synthesis delay. The structure index is one for the left source and rises at intervals of one with each source.

Figure 7 shows the effect of structure on delay. In order to obtain good astrometry, they also recommend that the source have an index of one at $2.3 \mathrm{GHz}$ also. Maps of the radio sources making up the ICRF may be found in Fey et al (1996 \& 1997b) and at http://www.usno.navy.mil. Figures 4, 6, and 7 show the complexity in spatial structure from a sample of extragalactic radio sources at $8.4 \mathrm{GHz}$. The sources in the ICRF are class 1 and 2.

Because the structure of these sources is variable in time, it is wise to measure their positions for apparent motions. Only those sources observed frequently over the past 25 years have sufficient history to yield excellent positions. A subset of these sources making up the ICRF has a large number of observations - these are the sources that were observed frequently on the geodetic programs. The southern hemisphere sources especially suffer from a lack of observations. In the future, some of the defining sources may become unsuitable. A maintenance program to obtain a large number of observations of the defining and candidate sources must be undertaken. 

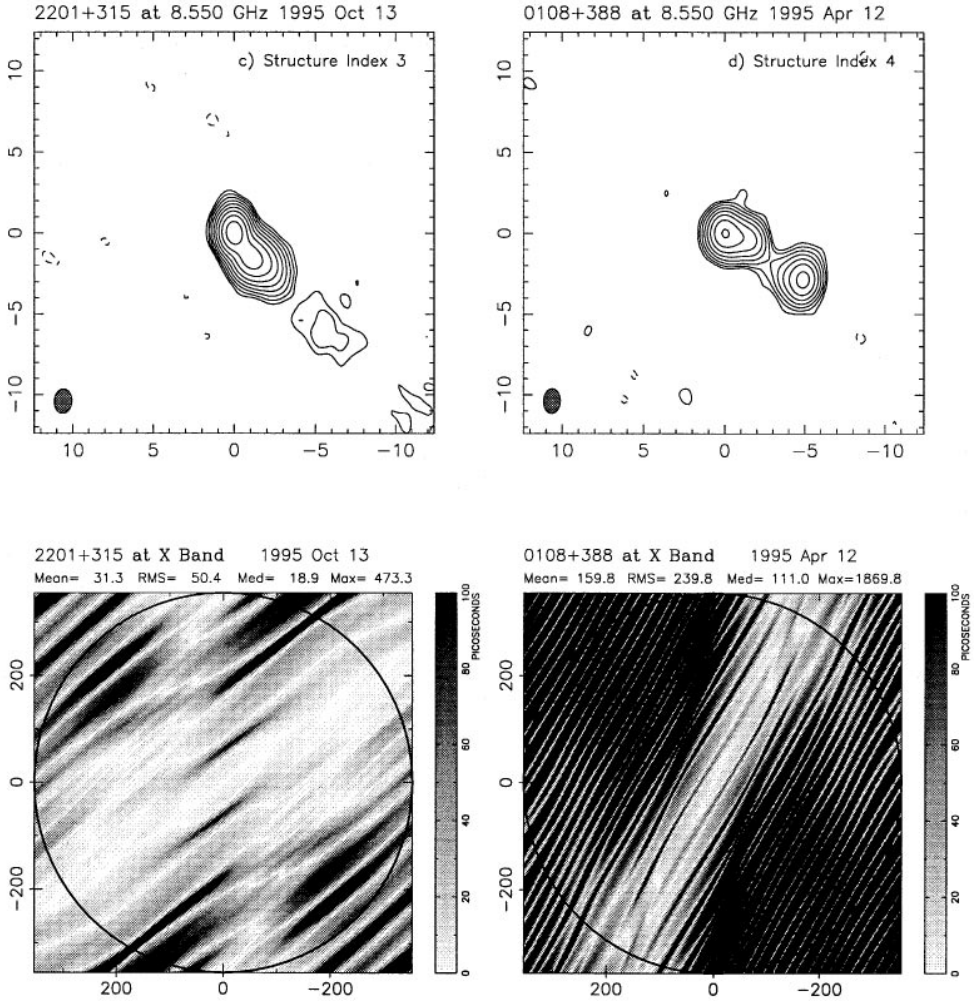

Figure 7 (Continued)

The geodetic programs will be a good source of a large number of observations of a subset of these sources. Astrometric maintenance observations will have to be made of a large number of sources using the available resources. This effort will require time on the VLBA, the EVN, MERLIN, and southern hemisphere telescopes in Australia, South Africa, and Brazil. Positional variation in the sources may be modeled if the variations in source structure are known. Charlot (1994) has shown that modeling the source structure in the complex source $3 \mathrm{C} 273$ significantly improves its positional stability.

Due to the lack of telescopes and land area in the southern hemisphere, it will be difficult to obtain observations of the source structure south of declination -20 degrees. The Highly Advanced Laboratory for Communication and Astronomy (HALCA) space mission may help this situation by obtaining observations for southern-hemisphere sources of a large number of spatial frequencies. Another method of monitoring these sources for changes in structure would be to monitor their flux density. There are no plans to do so in an organized way at this time.

Ma \& Shaffer (1991) have investigated using the geodetic database of the time varying positions for quasars. Ma \& Shaffer give limits of less than $50 \mu$ as/yr, while 
Eubanks et al (1995) find many sources with motions of order $30 \mu \mathrm{as} / \mathrm{yr}$. Many of these motions have not been confirmed (Fey et al 1997a). As already shown, apparent motions in the source 4C39.25 are attributable to variations in the spatial emission from the source (Fey et al 1997a). For well-behaved sources the positions are estimated to be repeatable at the level of 0.2 to 0.3 mas (Fey et al 1997a).

An estimate of the best stability in position of a compact radio source may be obtained from the numerous experiments made on close source pairs such as $1038+52$ A and B. For this pair of sources separated by 33 arcseconds, observations spanning a decade at wavelengths of 3.6 and $13 \mathrm{~cm}$ find changes in the angular separation of nearly 0.2 mas (Rioja et al 1997). These changes can be accounted for by motion of the reference feature in quasar B, which is a component of the jet displaying superluminal motion. The position stability of component A is estimated to be better than $10 \mu$ as.

\subsection{Limitations D ue to the Atmosphere, Scaling Factors and Models}

The propagation of signals in the troposphere causes one of the largest sources of measurement error. The error varies as a function of elevation and azimuth. Mapping functions that give the elevation dependence of tropospheric delay have reduced the systematic and random errors (Davis et al 1985, Herring 1992, Niell 1996). The effect of these azimuthal gradients is to cause a north-south asymmetry due to the greater troposphere thickness near the equator. Estimating these gradients reduces the radio source declinations by 0.5 mas at the equator (MacMillian $\&$ Ma 1997). In the future, mapping the troposphere via GPS satellites may give detailed models of the troposphere that will allow the delay path lengths in the atmosphere to be estimated with greater precision than at present from the VLBI data.

It is difficult to estimate the systematic errors introduced by the software used in the data reduction models. Comparison of different software routines such as GSFC's CALC and JPL's MODEST has recently shown discrepancies at the $5 \mu$ as level (Ma et al 1998). Previous analysis of geodetic data has shown that the formal errors in station coordinates should be multiplied by 1.5 (Ryan et al 1993). Ma et al (1998) have applied this to the errors in the positions of the defining sources. In addition, they have added 0.25 mas in quadrature in reporting the errors of the defining sources. Typical formal errors for sources in the ICRF are on the order of 0.1 mas. In the future a more complete understanding of the random and systematic errors should allow further refinement in the positions of quasars to levels well below 0.1 mas. The level at which variable source structure effects contribute significantly to these errors remains to be seen. For many sources, it is a significant effect at levels of a mas.

\subsection{Maintenance}

The IAU resolution adopting the ICRF also requested the IERS to provide its maintenance. The IERS VLBI coordinating center has been designated to carry out 
this task. It was also agreed at the IERS Directing Board meeting in San Francisco in 1998 that the ICRF be maintained by the VLBI coordinating center of the IERS. The level of observations for geodetic and earth orientation programs should increase over the next few years with the inception of NASA's Continuous Observation for Rotation of the Earth (CORE) program. The Mark IV correlator should come into operation in 1999, allowing larger arrays and more frequent observations needed by the CORE program. The U.S. Naval Observatory/NASA GSFC plan future observing programs to maintain the ICRF with yearly observations of the program sources. MERLIN also plans to contribute to this effort (Morrison et al 1997). The number of sources available for the reference frame will certainly increase. A VLBI survey of the nearly 2000 flat-spectrum sources from the Jodrell Bank-VLA Astrometric Survey is under way (Peck \& Beasley 1998).

\section{THE HIPPARCOSOPTICAL REFERENCE FRAME}

The extremely successful Hipparcos mission (1989-1993) established a global astrometric catalog that is not affected by atmospheric refraction and turbulence and is independent of the Earth orientation parameters. However, the construction of the consistent instrumental Hipparcos reference frame, the so-called sphere reconstruction problem, has a rank deficiency of 6. To align the Hipparcos system with the ICRF, two small rotations were determined, fixing the final orientation of the Hipparcos net at epoch 1991.25 and removing a global rotation from the proper motion. Because Hipparcos was not able to observe directly the optical counterparts of the defining sources of the ICRF, link procedures were adopted to achieve a posteriori adjustment (Lindegren \& Kovalevsky 1995).

The Hipparcos catalog contains 118,218 stars with typical precisions of 1.5 mas of the five astrometric parameters: position, parallax, and proper motion for the majority of the stars. Figure 8 shows the distribution in magnitudes of the catalog stars and the percentage of completeness as compared to the expected global galactic star counts in each magnitude interval. The Hipparcos catalog provides, for the first time at optical wavelengths, a global reference frame that is not affected by zonal and magnitude-dependent errors found in ground-based fundamental catalogs. However, the average star density of $2.7 \mathrm{stars} / \mathrm{deg}^{2}$ and the relatively bright magnitude of the catalog stars are not suitable for the direct adjustment of fainter objects to the ICRF in the small arcminute-sized fields of modern large telescopes. Hipparcos provides a high precision, first order net that can be extended by a secondary and much denser stellar net of fainter stars. This is currently achieved by new astrograph programs (Zacharias et al 1997) and eventually will be provided by future space missions.

In contrast to the extragalactic ICRF sources, the Hipparcos catalog stars display proper motions due to the angular component of the stars' space motion within the gravity field of our galaxy. As a consequence, the accuracies of the Hipparcos positions are strongly time dependent due to the proper motion error propagation. 


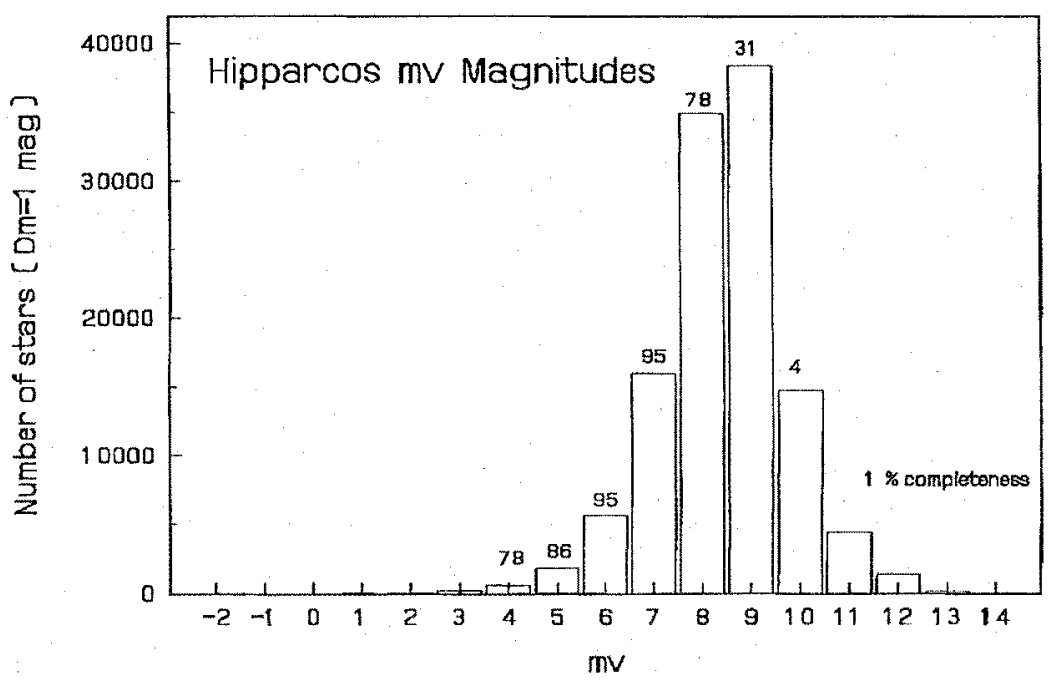

Figure 8 Number of Hipparcos stars versus visual magnitude. The completeness of the survey for each magnitude is shown at the top of the column. Note that Hipparcos is nearly complete to the eighth visual magnitude.

An additional smaller systematic contribution is due to the time-dependent error of the Hipparcos zero point adjustment (Figure 9). To preserve the high quality of the Hipparcos net at central epoch 1991.25 over an extended period, future space astrometry missions are indispensable. As an example, the proposed Deutsches Interferometer für Vielkanalphotometrie und Astrometrie (DIVA) (Roser et al 1997)

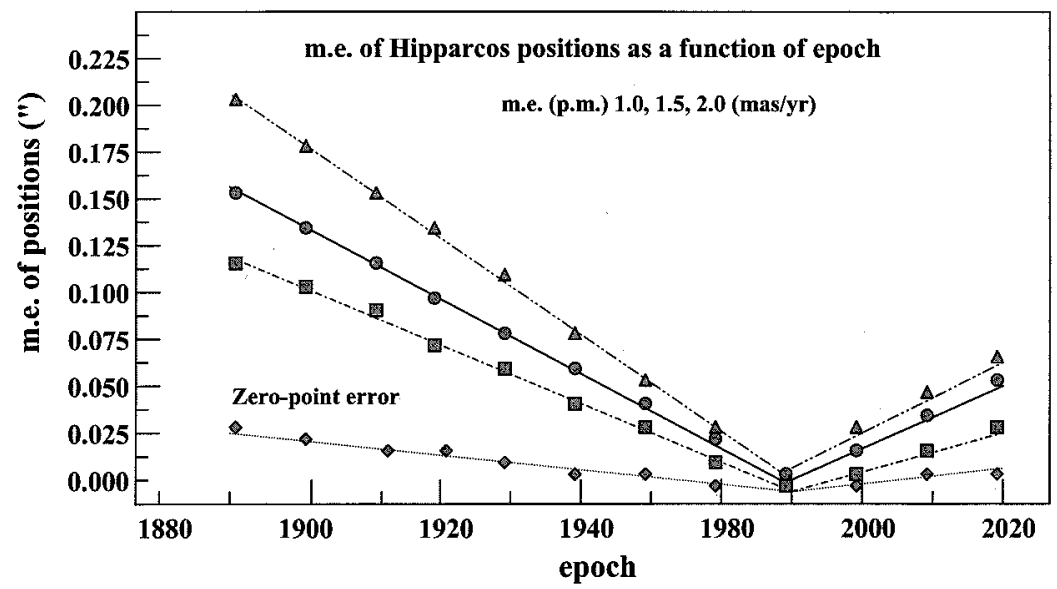

Figure 9 The accuracy of the Hipparcos positions as a function of time. The accuracy of the positions degrades with time because of imperfect knowledge of the proper motion of the stars. 
and Full-Sky Astrometric Mapping Explorer (FAME) (Johnston et al 1998) missions would limit the Hipparcos catalog error propagation to a few mas for many decades. The present Hipparcos extragalactic link can be improved further by re-observation and inclusion of additional radio stars with the VLBA, EVN, and MERLIN to extend the proper motion time basis.

\section{LINKING THE HIPPARCOS OPTICAL REFERENCE FRAME TO THE ICRF}

The Hipparcos mission's high-precision relative global stellar net of positions and proper motions had to be linked to the ICRF with similar precision. Because all suitable optical counterparts of the ICRF sources were too faint to be measured directly by Hipparcos (with the exception of 3C273B, but only with reduced accuracy), a direct link to the ICRF will be made only by future space missions that extend the magnitude range beyond that of Hipparcos.

The Hipparcos Extragalactic Link Working Group has used a variety of methods (Kovalevsky et al 1997), in particular VLBI, MERLIN, and VLA observations of selected radio stars that are optically bright Hipparcos catalog stars, and the direct measurement of ICRF optical counterparts. The most precise contribution has been obtained from long-term VLBI observations of 12 radio stars, which were tied to the ICRF using the closest ICRF source as the primary reference. From these long-term measurements the five astrometric parameters of these stars were determined to sub-mas precision (Lestrade et al 1995). A weighted least-squares solution using all of the methods was used to adjust the Hipparcos catalog to the ICRF with uncertainties of 0.6 mas and 0.25 mas/yr at epoch 1991.25 in the position and proper motion rotation parameters (Kovalevsky et al 1997).

\section{LINKING THE DYNAMICAL REFERENCE FRAME TO THE ICRF}

The advent of VLBI observations of spacecraft at planets and satellites has put the positions directly on the radio frame at accuracies of 1 to 3 mas. Further CCD observations of the planets using Hipparcos reference stars can also place the positions of planets and satellites at accuracies of 30 mas (Stone \& Dahn 1994). The major problem alluded to earlier is the transformation or alignment of the two different types of data onto a common reference frame, which is accomplished by taking VLBI measurements and solving for the rotation of the ephemeris that gives the best fit to the data. From a joint analysis of VLBI and LLR observations, a tie between the JPL planetary ephemerides and the IERS radio catalogs has been determined (Folkner et al 1994). The frame tie between the Hipparcos realization of the ICRF and the ICRF may be used to relate optical observations of the planets. Thus the present JPL ephemerides of the planets, DE405 and of the moon, LE405 (Standish et al 1995), use the reference frame of the ICRF. 


\section{REFERENCE FRAMESAT OTHER SPECTRAL RANGES}

The ICRF is defined at a radio frequency of $8.4 \mathrm{GHz}$. Care must be taken when extending this frame to other radio wavelengths. Most likely the nearby ICRF defining source will show little or no structure at higher radio frequencies, but at lower frequencies where the emission is self absorbed its position may change by the order of a mas or more. Other spectral ranges may be linked to the ICRF by use of link objects, which display emission at $8.4 \mathrm{GHz}$ and the spectral range. The Hipparcos realization of the ICRF also allows the frame to be transferred to other spectral ranges such as the IR through the IR counterparts of Hipparcos stars. The radio and optical regions of the spectrum both have frame ties to the IR. For example, Menten et al (1997) have determined the position of Sgr A* at IR wavelengths to 30 mas via the radio emission from $\mathrm{SiO}$ masers arising from the innermost parts of circumstellar envelopes of giant and supergiant stars that appear as compact IR sources.

\section{FUTURE IMPROVEMENTS IN REFERENCE FRAMES: Space and Ground Systems}

Hipparcos has demonstrated the feasibility of mas precision global astrometry from space. Follow-up missions aiming at an extension to much fainter limiting magnitudes and increased accuracy have been proposed. It should be noted that all these astrometry missions, including Hipparcos, primarily are directed to the solution of astrophysical problems-for example, galactic kinematics and the galactic distance scale from Cepheids and RR Lyras stars. To cover a substantial galactic volume and to obtain a complete sample of different stellar groups, therefore, the star selection may not be always optimal with regard to reference frame requirements.

In the future, reference frames at optical wavelengths will reach improved accuracies. Three proposed space missions, FAME (Johnston et al 1998), Global Astrometric Interferometer for Astrophysics (GAIA) (Gilmore et al 1998), and Space Interferometry Mission (SIM) (Shao 1998) propose to achieve global accuracies of 50, 4 , and $4 \mu$ as respectively. These missions will extend the accuracy of the optical reference frame by a factor of 20 to 250 .

FAME and GAIA are survey missions that will determine the positions of 40 to 500 million stars. The brighter stars will have positions at global accuracies of 50 and $4 \mu$ as respectively. To achieve this, the parallax and proper motions of these stars along with their orbital motions, if they are in multiple systems, must be measured to these accuracies. SIM (Shao 1998) is an integrating steerable space interferometer. The other missions are more or less based on modifications of Hipparcos' one-dimensional scanning principle with two superimposed fields of view separated by a constant basic angle of 60 to 100 degrees. Optical and detector technologies have improved significantly and all of these space missions will reach comparable or much higher accuracies than Hipparcos has reached, increasing the 
number of stars and magnitude range by orders of magnitude. A direct link to the ICRF optical counterparts can be made by all these missions, with SIM projected to reach the highest accuracy.

Improvement in the accuracy of wide-angle ground-based astrometric observations will be achieved by optical/IR interferometry. Hummel et al (1994) summarizes the results obtained with the Mount Wilson Interferometer. The Navy Prototype Optical Interferometer located near Flagstaff, Arizona is nearing completion. The astrometric accuracy of the instrument over wide angles should approach one mas (Johnston et al 1997). This instrument will be capable of measuring the positions of approximately 2000 bright Hipparcos stars and will be capable of maintaining the accuracy of the Hipparcos Frame in the northern hemisphere. The Keck Interferometer (Colavita et al 1998) may contribute to wide-angle astrometry although its primary function is 2-micron narrow-angle astrometry.

The premier accuracy of the ICRF will be challenged if FAME, GAIA, or SIM reach their goals. Frequent VLBI observations of the defining sources may allow their source structures to be modeled. Observations at radio frequencies higher than $8.4 \mathrm{GHz}$ may also reduce this effect because the structure may be dominated by a central compact source. Observations are under way at two $\mathrm{cm}$ with the VLBA (Eubanks, personal communication 1998). The models for the reduction of VLBI data in CALC/SOLVE and MODEST may be improved and further understood so that a factor of 1.5 does not need to be applied to the errors and systematic effects may become better understood. The atmospheric delay residuals pose the principal problem to improving the accuracy of VLBI astrometry. Figure 10 shows the formal errors in the ICRF solution. Future space VLBI missions with several elements in space can eliminate this source of error. A radio array in space will be very expensive. It would appear, however, that without a radio array in space the accuracy of VLBI astrometry over wide angles may be limited to more than $10 \mu$ as. Lensing may impose a limit of $10 \mu$ as as well.

\section{NEED FOR THE DEFINITION OF ASTRONOMICAL CONSTANTS AND TIME FOR THE REFERENCE SYSTEM}

The International Celestial Reference System was defined by the XXIII General Assembly of the IAU to take effect 1 January 1998. It is in accordance with the 1991 IAU recommendations that the origin be located at the solar system barycenter via modeling of VLBI observations in the framework of General Relativity. The pole is in the direction defined by the conventional IAU models for precession (Lieske et al 1977) and nutation (Seidelmann 1982) and the origin of right ascension is defined by fixing the right ascension of 3C273's (Hazard et al 1971) FK5 value transferred to J2000.0. The Hipparcos catalog contains all of the FK5 fundamental stars. Thus the location of the FK5 pole and origin of right ascension are related to an accuracy of a few mas. The coordinates of the mean pole at J2000 have been found from analysis of a long series of VLBI observations using a state-of-the-art 

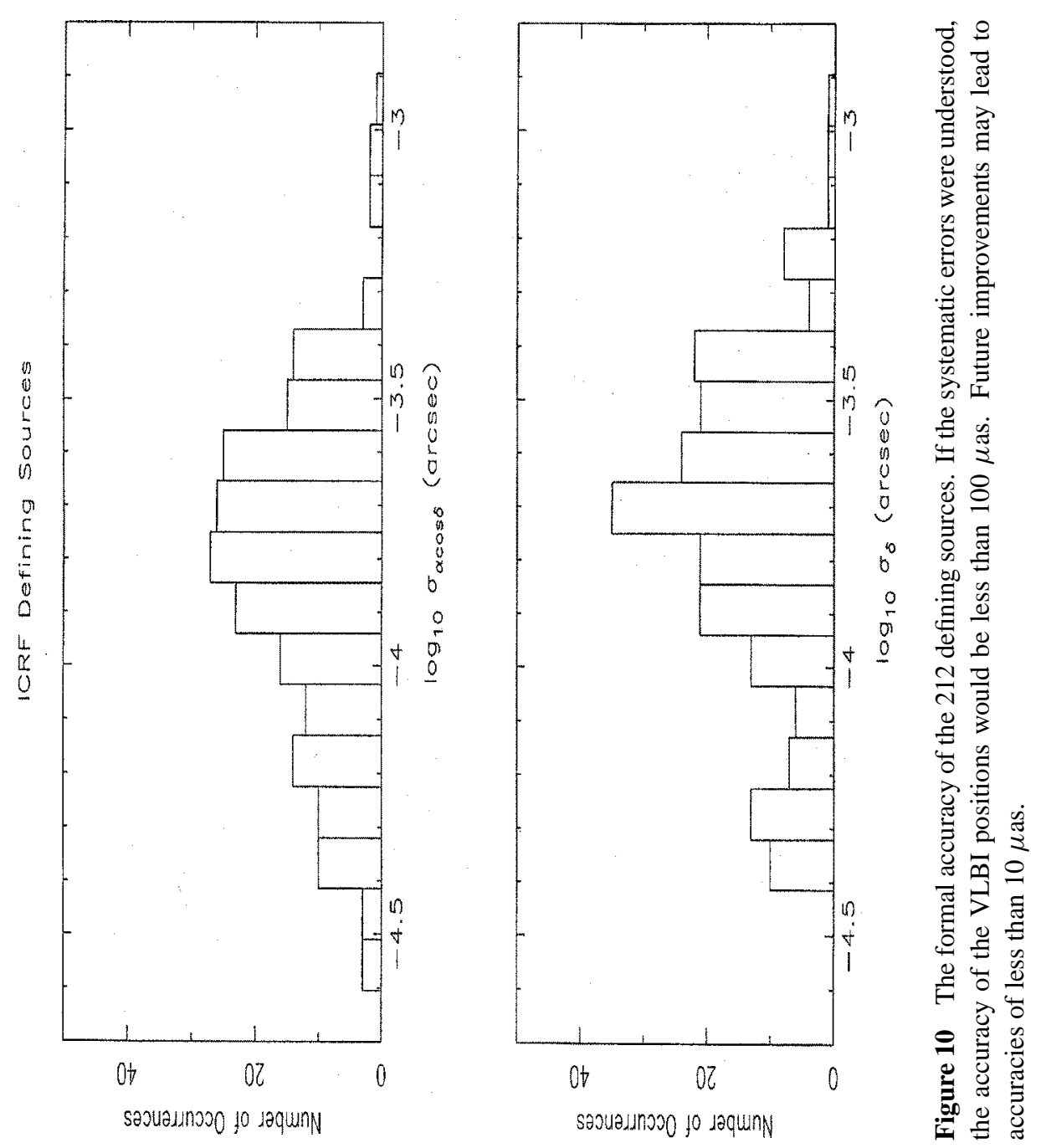
precession model to $17.3 \pm 0.2$ mas in the direction $12 \mathrm{~h}$ and $5.1 \pm 0.2$ mas in the direction of $18 \mathrm{~h}$ (IERS 1997).

McCarthy \& Luzum (1991) documented that the models for precession (Lieske et al 1997) and the IAU 1980 nutation theory (Seidelmann 1982) do not describe precisely the position of the pole. Ma et al (1998), in determining the positions of the ICRF sources, solve for an offset between the J2000 pole and the Celestial Ephemeris Pole of the ICRF in the form of celestial pole offsets angles in $\psi$ (longitude) and $\varepsilon$ (obliquity). The IERS conventions give improved values for these and other phenomena as well as constants such as the mass of the planets and gravitational constant. The present status of IAU standards is given in Fukushima (1997). There is no physical theory to adequately describe many of the phenomena at the level of precision needed. The IERS conventional model for nutation is precise at the 0.1 mas level. Therefore a numerical fit to the data may have to suffice to describe these phenomenon at the $10-1 \mu$ as level. New constants for precession and other effects, as well as models for nutation and other phenomena, must be adopted by the IAU. The adoption of the nonrotating origin (Guinot 1979, Captaine et al 1986) would simplify future definitions of precession and models for nutation.

There is also the problem with the definition of time. Special Relativity dictates that there is no independent order of events that is universal at all locations. Therefore the coordinate frame must be looked upon as a four-dimensional space. The position of the Earth-moon barycenter is looked upon as a quasi-inertial space because it contains a "weak" gravitational field in which the effects of General Relativity will be small. Transformations from an Earth-centered geocentric coordinate time frame to the barycentric coordinate time are given in the IERS Conventions.

\section{CONCLUSIONS}

The ICRF has been established using the positions of 212 extragalactic radio sources whose positions are accurate to one mas with the majority being less than 0.5 mas. The Hipparcos frame is the realization of this frame at optical wavelengths. This has come about as a result of the accuracies achieved by VLBI radio astrometry and the characteristics of the emitting sources. The ICRF establishes the ICRS. However, the definition of the ICRS needs to be refined by updating astronomical constants and models in time, precession, etc to take advantage of this increase in accuracy. This should be accomplished at the next IAU meeting in 2000. Further increases in measurement precision to $4 \mu$ as from space missions at optical wavelengths may dictate a redefinition of the ICRF, accompanied by improvement in constants and models for the ICRS.

\section{ACKNOWLEDGMENTS}

The authors would like to thank PK Seidelmann, DD McCarthy and A Fey for critical reading of the manuscript, A Fey for assistance with the figures, and L Treadway for preparing the manuscript. 


\section{Visit the Annual Reviews home page at http://www.AnnualReviews.org}

\section{LITERATURE CITED}

Aoki S, Guinot B, Kaplan GH, Kinoshita H, McCarthy DD, et al. 1982. Astron. Astrophys. 105:359-61

Allen LR, Anderson B, Conway RG, Palmer HP, Reddish VC, et al. 1962a. MNRAS 125: 477-99

Allen LR, Hanburry-Brown R, Palmer HP. 1962b. MNRAS 125:57-74

Appenzeller I. 1994. Highlights of Astronomy: XXII IAU Gen. Assem. Vol 10. Dordrecht: Kluwer

Argue AN, de Vegt C, Elsmore B, Fanselow J, Harrington R, et al. 1984. Astron. Astrophys. 130:191-99

Arias EF, Charlot P, Feissel M, Lestrade JF. 1995. Astron. Astrophys. 303:604-08

Arias F, Lestrade JF, Boucher C, Feissel M. 1991. Astrophys. Space Sci. 177:187-92

Auwers A. 1879. Pub. Astr. Ges. p. 14

Baade W, Minkowski R. 1954. Ap. J. 119:20614

Bare C, Clark BG, Kellermann KI, Cohen MH, Jauncey DL. 1967. Science. 157:189-91

Bergeron J, ed. 1992. Highlights of Astronomy. XXI IAU Gen. Assem., Vol. 9. Dordrecht: Kluwer

Bergeron J, ed. 1997. Highlights of Astronomy. XXIII IAU Gen. Assem., Vol. 10. Dordrecht: Kluwer

Bolton JG, Stanley GJ. 1948. Aust. J. Sci. Res. Ser. A. 1:58-69

Bross B. 1937. General Catalog of 33,342 Stars for the Epoch 1950. Wash. DC: Carnegie Inst. Wash.

Brosche P, Wade CM, Hjellming RM. 1973. Ap. J. 183:805-18

Broten NW, Legg TH, Locke JL, McLeish CW, Richards RS et al. 1967. Nature. 215: 38

Capitaine N, Guinot B, Souchay J. 1986. Celest. Mech. 39:283-307

Carr TD, May J, Olson CN, Walls GF. 1965. IEEE NEREM Rec. 7:222
Charlot P. 1994. Proc. VLBI TechnologyProgress and Future Observational Possibilities, Kyoto, Japan (September 1993), ed. T Sasso, S Manabe, O Kameya, M Inoue, p. 287. Tokyo: Terra Sci. Publ. Co.

Clark TA, Corey BE, Davis JL, Elgered G, Herring TA, et al. 1985. IEEE Trans. Geosci. Remote Sens. GE-23:438

Clark TA, Hutton LK, Marandino GE, Counselman CC, Robertson DS, et al. 1976. Astron. J. 84:599-603

Cohen MH, Shaffer DB. 1971. Astron. J. Let. 76:91-100

Colavita MM, Boden AF, Crawford SL, Meinel AB, Shao M. 1998. Proc. SPIE 3350. pp. $776-84$

Counselman CC III. 1978. Annu. Rev. Astron. Astrophys. 14:197-214

Davis JL, Herring TA, Shapiro II, Rogers AEE, Elgered G. 1985. Radio Sci. 20:15931607

Eichhorn H. 1974. Astronomy of Star Positions: A Critical Investigation of Star Catalogues, the Methods of their Construction, and their Purpose. New York: Ungar

Eubanks TM, Matsakis DN, Josties FJ, Archinal BA, et al. 1995. Proc IAU Symp. 166, ed. E. Hog, PK Seidelmann. Dordrecht: Kluwer Academic

European Space Agency. 1997. The Hipparcos and Tycho Catalogs. sci. coord. MAC Perryman, ESA SP-1200

Fanselow JK, Sovers OJ, Thomas JB, Purcell GH, Cohen E et al. 1984. Astron. J. 89:98798

Ferraz-Mello S, Morando B, Arlot J-E, eds. 1995. IAU Symp. No. 172, Dynamics, Ephemerides and Astrometry of the Solar System. Dordrecht: Kluwer

Fey AL, Charlot P. 1997b. Ap. J. Suppl. 111:95142

Fey AL, Clegg AW, Fomalont EB. 1996. Ap. J. Suppl. 105:299-330 
Fey AL, Eubanks TM, Kingham KA. 1997a. Astron. J. 114:2284-91

Fey AL, et al. 1992. Astron. J. 104:891-96

Fey AL, Russell JL, de Vegt C, Zacharias N, Johnston KJ, et al. 1994. Astron. J. 107:38591

Folkner WM, Charlot P, Finger MH, Williams JG, Sovers OJ, et al. 1994. Astron. Astrophys. 287:279-89

Fricke W. 1981. Definition and practical realization of the reference frame in the FK5the role of planetary dynamics and stellar kinematics in the definition. In Reference Coordinate Systems for Earth Dynamics: Proceedings of the $56^{\text {th }}$ Colloquium of the IAU, Warsaw, Poland, ed. EM Gapschkin, B Kolaczek, Astrophys. Space Sci. Lib. Proc. Dordrecht: Kluwer

Fricke W. 1982. Astron. Astrophys. 107:L13-16

Fricke W, Kopff A. 1963. FK4 Veroffentl. Astron. Rechen-Institut. Heidelberg. No 10. Germany: G. Braun, Karlsruhe

Fricke W, Schwan H, Corbin T. 1991. FK5 II Extension. Veroffentl. Astron. Rechen-Institut. Heidelberg. Vol 33. Germany: G. Braun, Karlsruhe

Fricke W, Schwan H, Lederle T. 1988. FK5 I Veroffentl. Astron. Rechen-Institut. Heidelberg. Vol 32. Germany: G Braun, Karlsruhe

Fukushima T. 1997. IAU Symp. 172, Dynamics, Ephemerides and Astrometry of the Solar System. pp. 461-68

Gilmore G, Perryman M, Lindegren L, Favata F, Hoeg E. 1998. Proc. SPIE 3350. pp. 54150

Gordon D. 1985. NASA Intern. Memo. Greenbelt, MD: Goddard Space Flight Center

Guinot B. 1979. IAU Symposium No. 82, Time and the Earth's Rotation, ed. DD McCarthy, JD Pilkington, pp. 7-18. Dordrecht: D. Reidel Publ. Co.

Guirado JC, et al. 1995. Astron. J. 110:2586-96

Hazard C, Sutton J, Argue AN, Kenworthy CM, Morrison LV, Murray CA. 1971. Nature. 233:89-91

Herring TA. 1992. In Symposium on Refraction of Transatmospheric Signals in Geodesy, ed.
JC De Munck, TA Spoelstra, p. 157. Delft: Netherlands Geod. Comm.

Hjellming RM, Bignell RC, 1982. Science. 216:1279-85

Hog E, Seidelmann PK, eds. 1995. IAU Symp.

No. 166, Astronomical and Astrophysical Objectives of Sub-Milliarcsecond Astrometry. Dordrecht: Kluwer

Hogg DE, Macdonald GH, Conway RG, Wade CM. 1969. Astron. J. 74:1206-13

Hughes JA, Smith CA, Kaplan GH, eds. 1991. Proc. IAU Coll. 127, Reference Systems. Wash. DC: USNO

Hummel CA, Mozurkewich D, Elias N, Quirrenbach A, Buscher DF, et al. 1994. Astron. J. 108:326-36

International Earth Rotation Service. 1996. IERS Conv. (1996), IERS Tech. Note 21, ed. DD McCarthy. Paris: Obs. Paris

International Earth Rotation Service. 1997. IERS Annu. Rep. (1996). Paris: Obs. Paris

International Earth Rotation Service. 1997. Tech. Note 23. Paris: Obs. Paris

Johnston KJ, Fey AL, Zacharias N, Russell JL, Ma C, et al. 1995. Astron. J. 110:880915

Johnston KJ, Hutter DF, Benson JA, Elias $\mathrm{N}$, Armstrong JT, et al. 1997. Proc. IAU Symp.189, Fundamental Stellar Properties. ed. T Bedding, A Booth, J Davis, pp. 39-42. Dordrecht: Kluwer

Johnston KJ, Russell J, de Vegt C, Hughes J, Jauncey DL, et al. 1988. IAU Symp. 129, The Impact of VLBI on Astrophysics and Geophysics, ed. M Reid, J Moran, pp. 317-18. Dordrecht: Reidel

Johnston KJ, Russell JL, de Vegt C, Zacharias N, Hindsley R, et al. 1991. Proc of IAU Coll. No. 127, ed. J Hughes, CA Smith, GH Kaplan, pp. 123-29. Wash. DC: USNO

Johnston KJ, Seidelmann PK, Reasonberg RD, Phillips JD, Horner S, et al. 1998. Pap.USNO, Wash. DC: USNO

Kopff A. 1937. Dritter Fundamental-Katalog Part I Veroffentl. Astron. Rechen-Institut. zu Berlin-Dahlem No 54. Berlin: Ferd. Dummlers 
Kovalevsky J. 1998. Annu. Rev. Astron. Astrophys. 36:99-129

Kovalevsky J, Lindegren L, Perryman MAC, Hemenway PD, Johnston KJ, et al. 1997. Astron. Astrophys. 323:620-33

Kovalevsky J, Mueller II. 1981. Proc. IAU Coll. 56

Kovalevsky J, Mueller II, Kolaczek B. 1989. Reference Frames in Astronomy and Geophysics. APSC. Lib. 154. Dordrecht: Kluwer

Lestrade JF, Jones DL, Preston RA, Phillips RB, Titus MA. 1995. Astron. Astrophys. 304:18288

Lieske JH, Lederle T, Fricke W, Morando B. 1977. Astron. Astrophys. 58:1-16

Lindegren L, Kovalevsky J. 1995. Astron. Astrophys. 304:189-201

Ma C. 1978. PhD dissertation. NASA TM 79582. Greenbelt, MD: Goddard Space Flight Center

Ma C, Arias EF, Eubanks TM, Fey AL, Gontier AM, et al. 1998. Astron. J. 116:516-46

Ma C, Clark TA, Ryan JW, Herring TA, Shapiro II, et al. 1986. Astron. J. 92:1020-29

Ma C, Shaffer DB, de Vegt C, Johnston KJ, Russell JL. 1990. Astron. J. 99:1284-98

Ma C, Shaffer DB. 1991. IAU Coll. 127, ed. JA Hughes, CA Smith, GH Kaplan, pp. 135-44. Wash. DC: USNO

MacMillan DS, Ma C. 1997. Geophys. Res. Lett. 24:453-56

Matthews TA, Sandage A. 1963. Ap. J. 138: 30-56

McCarthy DD, Luzum BJ. 1991. Astron. J. 102: 1889-95

Meeks ML. 1976. Ed. Astrophysics: Radio Observations, Vol. 12, Part C, Methods of Experimental Physics, pp. 531-69. New York: Academic Press

Melbourne W, Anderle R, Feissel M, King R, McCarthy D, et al. 1983. USNO Circ. 167. Wash. DC: USNO

Menten KM, Reid MJ, Eckart A, Genzel R. 1997. Ap. J. Lett. 475:111-14

Mignard F, Froeschle M. 1997. Proc. ESA Symp. Hipparcos-Venice 1997. ESA SP-402. pp. $57-60$
Monet DG. 1988. Annu. Rev. Astron. Astrophys. 26:413-40

Morabito DD, Niell AE, Preston RA, Linfield RP, Wehrle AE, Faulkner J. 1986a. Astron. J. 91:1038-50

Morabito DD, Preston RA, Linfield RP, Slade MA, Jauncey DL. 1986b. Astron. J. 92:54651

Moran JM, Crowther PP, Burke BF, Barrett AH, Rogers AEE, et al. 1967. Science 157:676-77

Morrison LV, Garrington ST, Argyle RW, Davis RJ. 1997. Proc. ESA Symp. Hipparcos-Venice 1997. ESA SP-402. pp. 143-45

Mueller II. 1969. Spherical and Practical Astronomy. New York: F Ungar Publ.

Newcomb S. 1905a. Astronomical Papers of the American Ephemeris and Nautical Almanac. Vol 8, Part 2. Wash. DC: Nautical Almanac Office, USNO

Newcomb S. 1905b. Astronomical Papers of the American Ephemeris and Nautical Almanac. Vol 8, Part 1. Wash. DC: Nautical Almanac Office, USNO

Niell AE. 1996. J. Geophys. Res. 101(B2): 3227-46

Oke JB. 1963. Nature 197:1040-41

Palmer HP. 1962. IAU Symposium No. 15, Problems of Extragalactic Research, ed. GC McVittie, pp.315-25. New York: Macmillan

Peck AB, Beasley AJ. 1998. IAU Coll. 164, ed. JA Zensus, GB Taylor, JM Wrobel. ASP Conf. Ser. Vol. 144:155-56

Reynolds JE, Jauncey DL, Russell JL, King EA, McCulloch PM, et al. 1994. Astron. J. 108:725-30

Rioja MJ, Marcaide JM, Elodegui P, Shapiro II. 1997. Astron. Astrophys. 325:383-90

Robertson DS. 1975. Geodetic and Astrometric Measurements with Very Long Baseline Interferometry. $\mathrm{PhD}$ thesis. MIT

Robertson DS, Fallon FW, Carter WE. 1986. Astron. J. 91:1456-62

Rogers AEE. 1970. Radio Sci. 5:1239-47

Rogers AEE, Cappallo RJ, Hinteregger HF, Levine JL, Nesman EF, et al. 1983. Science. 219:51-53

Roser S, Bastian U, de Boer KS, Hog E, Roser 
HP, et al. 1997. Proc. ESA Symp. HipparcosVenice 1997. ESA-SP 402. pp. 777-82

Russell JL, Johnston KJ, Ma C, Shaffer D, de Vegt C. 1991. Astron. J. 101:2266-73

Russell JL, et al. 1992. Astron. J. 103:2090-98 Russell JL, et al. 1994. Astron. J. 107:379-84

Ryan JW, Ma C, Caprette DS. 1993. NASA Tech. Mem. 104572. Greenbelt, MD: NASA

Ryle M, Smith FG. 1948. Nature 162:462-63

Ryle M. 1972. Nature 239:435-38

Schmidt M. 1963. Nature 197:1040

Seidelmann PK. 1982. Celest. Mech. 27:79-106

Seidelmann PK. 1992. Ed. Explanatory Supplement to the Astronomical Almanac. Mill Valley, CA: Univ. Sci. Books

Shao M. 1998. Proc. SPIE 3350. pp. 536-40

Soffel MH. 1989. Relativity in Astrometry, Celestial Mechanics, and Geodesy. Berlin: Springer-Verlag

Sovers OJ, Edwards CD, Jacobs CS, Lanyi KM, Liewer KM, Treuhaft RN. 1988. Astron. J. 95:1647-58

Sovers OJ, Fanselow JL. 1987. JPL Publ. 83-39 Rev. 3. Pasadena: Jet Propulsion Laboratory Sovers OJ, Fanselow JL, Jacobs CS. 1998. Rev. Mod. Phys. 70:1393-1454

Sovers OJ, Ma C. 1985. EOS. 66:858 (Abstr.)

Standish EM. 1981. Astron. Astrophys. 101: L17-L18

Standish EM. 1982. Astron. Astrophys. 114: 297-302

Standish EM, Newhall XX, Williams JG,
Folkner WF. 1995. JPL IOM. 314:10-127

Stone RC, Dahn CC. 1995. IAU Symp. 166, Astronomical and Astrophysical Objectives of Sub-milliarcsecond Astrometry, ed. E Hog, PK Seidelmann, P Dordrecht. pp. 3-8. Dordrecht: Kluwer

Thompson AR, Moran JM, Swenson GW Jr. 1986. Interferometry and Synthesis in Radio Astronomy. New York: Wiley

van Altena WF. 1983. Annu. Rev. Astron. Astrophys. 21:131-64

Vondrak J, Capitaine N. 1998. Journees 1997 , Reference Systems and Frames in the Space Era, Present and Future Astrometric Programs. Paris: Obs. Paris

Wade CM. 1970. Ap. J. 162:381-90

Wade CM, Johnston KJ. 1977. Astron. J. 82: 791-95

Wielen, et al. 1997. Proc. ESA Symp. Hipparcos-Venice 1997. ESA SP-402. pp. 727-32

Woolard EW, Clemence GM. 1966. Spherical Astronomy. New York: Academic Press

Wytrzyszczak IM, Lieske JH, Feldman RA, eds. 1996. Ed. IAU Coll. No. 165, Dynamics and Astrometry of Natural and Artificial Celestial Bodies. Dordrecht: Kluwer

Zacharias N, de Vegt C, Murray CA. 1997. Proc. ESA Symp. Hipparcos-Venice 1997. ESA SP402. pp. $177-80$

Zensus JA, Diamond PJ, Napier PJ. 1995. Very Long Baseline Interferometry and the VLBA. ASP Conf. Proc. 82. San Francisco: ASP 